\title{
Miquel Crusafont, George Simpson y la internacionalización de los estudios de paleontología evolutiva en España
}

\author{
Jesús I. Catalá Gorgues (*) \\ $\left({ }^{*}\right)$ Departamento de Humanidades. CEU, Universidad Cardenal Herrera \\ jicatala@uch.ceu.es
}

SUMARIO: 1.-Introducción. 2.-Los inicios de la relación. 3.-Orientaciones y divergencias. 4.--La proyección internacional. 5.-Conclusión.

RESUMEN: El paleontólogo catalán Miquel Crusafont Pairó (1910-1983) fue un destacado especialista en los mamíferos fósiles del Terciario de la Península Ibérica. Además, fue el introductor de nuevos enfoques en torno a la biología evolutiva en España. A pesar de las numerosas dificultades que había para investigar en evolución en la España franquista, Crusafont logró alcanzar una posición notable en la comunidad internacional de paleontólogos. Aparte de ser un científico competente, se vio favorecido por la amistad del paleontólogo estadounidense George Gaylord Simpson (1902-1984), uno de los impulsores de la síntesis evolucionista. Este artículo se ocupa de la relación entre ambos científicos y enfatiza el papel fundamental que Simpson representó en la internacionalización del trabajo de Crusafont.

PALABRAS CLAVE: evolución, paleontología, biometría, España, siglo XX.

KEY WORDS: Evolution, Palaeontology, Biometry, Spain, 20th century.

\section{Introducción $(*)$}

La relación científica y personal entre el paleontólogo catalán Miquel Crusafont Pairó (1910-1983) y el profesor estadounidense George Gaylord Simpson (1902-1984), uno de los principales adalides de la nueva síntesis evolucionista, es un aspecto central para la historia de la paleontología

(*) Este trabajo es resultado del proyecto «Los años grises. La investigación biológica en la España del primer franquismo» (MICIN, I+D HAR 2010-21333C01). Una versión previa fue presentada en el simposio «Naturaleza y laboratorio» (Sanlúcar de Barrameda, 3-4 de septiembre de 2011). 
evolutiva en la España franquista. Iniciada en una época, los años cuarenta, en la que las circunstancias sociopolíticas habían vuelto a tornarse bien difíciles para todo lo que oliera a evolucionismo, su mantenimiento a lo largo de cuatro decenios la confirma como caso de estudio obligado. La relación ha quedado registrada con detalle en una larga serie de cartas, depositadas en el Institut Català de Paleontologia de Catalunya Miquel Crusafont y en la American Philosophical Society, que constituyen la fuente principal para este trabajo.

Crusafont, que se inició muy joven en la investigación paleontológica (antes incluso de ingresar en la universidad para estudiar la carrera de Farmacia), ligó su labor científica al Museo de Sabadell, fundado en 1931 y del cual él mismo, así como su padre, fue impulsor. En dicho centro se constituyó en 1934 una sección de paleontología y geología. Por esa misma época, inició su colaboración de más de dos décadas con otro joven científico, Josep Fernández de Villalta Comella (1913-2003). Sus artículos conjuntos de 1933 y 1934 en el Butlletí de la Institució Catalana d'Història Natural marcaron el arranque de una de las líneas maestras de investigación de nuestro personaje: el estudio de los vertebrados fósiles, concretamente del Mioceno de Cataluña, ampliado después a otras series del Terciario ibérico y aun del Cuaternario ${ }^{1}$.

Tras la Guerra Civil, y una vez superada, gracias a que se sobreseyó su causa, la dramática circunstancia de ser apresado, acusado y juzgado de manera sumarísima por catalanista por las autoridades del nuevo régimen, Crusafont se incorporó al Museo de Sabadell como subdirector ${ }^{2}$. Fue en tal contexto institucional donde empezó a relacionarse epistolarmente con importantes autores extranjeros, entre otros, George G. Simpson.

La relación de Simpson con Crusafont ha sido glosada e incluso tratada con cierta extensión por diferentes autores que se han aproximado a la vida y obra del segundo. Así, su discípulo Jaume Truyols ${ }^{3}$ y su biógrafa

1. Mañosa, Montserrat. Miquel Crusafont i Pairó, Sabadell, 1910-1983. L'escola paleontològica de Sabadell. In: Camarasa, Josep Maria; Roca, Antoni, dirs. Ciència i tècnica als Països Catalans: una aproximació biográfica. Barcelona: Fundació Catalana per a la Recerca; 1995, p. 14431472, especialmente p. 1448-1449.

2. Mañosa, n. 1, p. 1452-1453. Para más detalles sobre dicho contexto, véase el artículo de Acosta, en este mismo monográfico de Dynamis.

3. Truyols Santonja, Jaume. L'obra científica del Doctor Miquel Crusafont i Pairó. Butlletí de la Institució Catalana d'Història Natural. 1986; 53: 19-36. 
Montserrat Mañosa ${ }^{4}$, han puesto de relieve la importancia de la misma. Más detallados han sido los estudios de Jordi Agustí ${ }^{5}$ y, especialmente, de Thomas F. Glick ${ }^{6}$, específicamente dedicado este último a la propia relación que aquí de nuevo nos ocupa. Por el contrario, los estudiosos de Simpson le han dado escaso relieve, hasta el punto que su principal biógrafo, Léo F. Laporte $^{7}$, ni siquiera hace mención del autor catalán.

En este trabajo, vamos a procurar aportar nuevos detalles al conocimiento de la relación entre ambos personajes y vamos a centrar la parte principal de nuestro análisis en la función, a nuestro entender crucial, que desarrolló Simpson en la conquista de una proyección internacional para Crusafont, en lo que supuso al mismo tiempo la internacionalización de los estudios de paleontología evolucionista desarrollados en la España franquista. Debe señalarse que el resultado de la Guerra Civil trajo una reactivación de las tendencias antievolucionistas en España durante los años cuarenta, lo cual no impidió que los ecos de los desarrollos de la teoría sintética de la evolución llegaran también al país por esa misma época. Así, algunas aportaciones del propio Simpson no tardaron en ser conocidas y valoradas, aunque no necesariamente aceptadas ${ }^{8}$. El punto de partida de la relación de los protagonistas de este trabajo se sitúa, pues, en un tiempo en que las condiciones no eran precisamente ideales para el libre debate de la teorías evolucionistas en España. Solamente bajo la asunción de un evolucionismo teísta fue posible, desde finales de los cuarenta, publicar opiniones alternativas al puro antievolucionismo ${ }^{9}$. En todo caso, Crusafont, católico convencido, no se sintió incómodo con ello.

\footnotetext{
4. Mañosa, n. 1.

5. Agustí, Jordi. L'evolucionisme meridional de Miquel Crusafont. Arraona. 1994; 14: 29-43.

6. Glick, Thomas F. Miquel Crusafont i George Gaylord Simpson: interferències biogràfiques, confluències històriques. Arraona. 1994; 14: 45-56. Este artículo aparece traducido al castellano en Glick, Thomas F. Darwin en España. 2a ed. Valencia: Universitat de València; 2010, p. 104113. Véase además Glick, Thomas F. Miquel Crusafont e a introduição da teoria sintética da evolução na Espanha. In: Bertol Domingues, Heloisa; Romero Sá, Magali; Puig-Samper, Miguel Ángel; Ruiz Gutiérrez, Rosaura, eds. Darwinismo, meio ambiente, sociedade. São Paulo / Rio de Janeiro: Via Lettera / MAST; 2009, p. 221-235.

7. Laporte, Léo F. George Gaylord Simpson: paleontologist and evolutionist. New York: Columbia University Press; 2000.

8. Pelayo, Francisco. Debatiendo sobre Darwin en España: antidarwinismo, teorías evolucionistas alternativas y síntesis moderna. Asclepio. 2009; 61: 101-128.

9. Blázquez, Francisco. La recepción del evolucionismo en la universidad española (1939-1999). Anuario de Historia de la Iglesia. 2009; 18: 55-68. Blázquez, Francisco. A Dios por la ciencia. Teología natural en el franquismo. Asclepio. 2011; 63: 453-476.
} 


\section{Los inicios de la relación}

El origen de la relación entre Simpson y Crusafont no tiene nada de extraordinario. Los usuales intercambios de publicaciones entre especialistas fueron el punto de arranque, allá por el convulso final de la primera mitad de la década de los cuarenta. La conflagración mundial condicionaba, desde luego, el tránsito epistolar entre nuestros personajes. El 24 de abril de 1945, Crusafont y Villalta escribían a Simpson para anunciarle que, por correo aparte, le enviaban «a parcel with "separata" of our new works on Spanish Paleomastology», al tiempo que le solicitaban el envío de trabajos recientes sobre faunas de mamíferos fósiles del Viejo Mundo; además, mencionaban cómo la situación bélica impedía un contacto más fluido con el American Museum of Natural History, donde Simpson trabajaba ${ }^{10}$. Este no contestó hasta el día 5 de junio ${ }^{11}$.

Las 56 publicaciones que a su vez envió Simpson a Sabadell sí alcanzaron su destino avanzado el verano. En la difícil situación de la España de la posguerra civil, era muy difícil acceder a la literatura científica más reciente; de ahí que Crusafont expresara su profundo agradecimiento e incluso se atreviera a pedir algún trabajo más, a cambio de mantener constantemente surtido a Simpson de publicaciones propias «and offering to you for anything you wish $(\text { sic })_{»}{ }^{12}$. Éste, por su parte, tras dejar pasar unos meses y ponerse en la lectura de las publicaciones de Crusafont y Villalta - Simpson sabía leer castellano-, las ponderó como «contributions to science of the highest quality» ${ }^{13}$.

Simpson, seguramente, no se estaba limitando a hacer un cumplido y pensaba con sinceridad aquello que decía. Tal vez no habría esperado, a priori, que de la postrada España franquista pudieran salir trabajos paleontológicos realmente interesantes. Pero el caso es que allí tenía aquellas separatas, que probaban el talento para el estudio de los mamíferos fósiles

10. Crusafont, Miquel; Fernández de Villalta, José. Copia de Carta a George G. Simpson. 24 Abr 1945. Arxiu Miquel Crusafont (en adelante AMC), doc. 065a/Cacad/8843. El archivo está depositado en el Institut Català de Paleontologia Miquel Crusafont, en Sabadell.

11. Simpson, George G. Carta a Miquel Crusafont y José Fernández de Villalta. 5 Jun 1945. AMC, doc. $065 \mathrm{a} / \mathrm{Cacad} / 8848$.

12. Crusafont, Miquel; Fernández de Villalta, José. Copia de Carta a George G. Simpson. 21 Ago 1945. AMC, doc. 065a/Cacad/8866.

13. Simpson, George G. Carta a Miquel Crusafont. 2 Nov 1945. AMC, doc. 065a/Cacad/8884. 
de Crusafont y la incipiente conformación de un núcleo de práctica naturalista a su alrededor.

Durante el año 1946, el intercambio epistolar y de publicaciones se fue consolidando. Crusafont optó, eso sí, por escribir en francés, disculpándose por su pobre inglés escrito. Para entonces, estaba realizando estudios sobre los castores fósiles de España, en los cuales Crusafont pensaba aplicar protocolos biométricos desarrollados por Simpson ${ }^{14}$. El largo artículo correspondiente, que apareció firmado conjuntamente con Villalta y el veterano paleontólogo y sacerdote José Ramón Bataller (1890-1962), supuso el primer estudio de paleontología biométrica publicado en España. A pesar de esa autoría conjunta, es de notar que la parte estrictamente biométrica fue de exclusiva responsabilidad de Crusafont, como se hacía constar de forma explícita en la propia publicación, mientras que de los tres firmantes fue la responsabilidad de la parte descriptiva sistemática ${ }^{15}$. Crusafont tomó como referencia principal para la parte biométrica Quantitative zoology ${ }^{16}$, libro escrito por Simpson y su esposa, Anne Roe (1904-1991), que marcó la influencia primordial del norteamericano en el catalán ${ }^{17}$.

Aquel año de 1946 también fue el de la publicación de un resonante descubrimiento por parte de Villalta y Crusafont, el de un nuevo género de mamíferos artiodáctilos del Mioceno, al que denominaron Triceromeryx. Los materiales originales sobre los que se basó la descripción procedían del yacimiento de la Hidroeléctrica, en la ciudad de Madrid, que había sido explorado por Eduardo Hernández-Pacheco (1872-1965) un cuarto de siglo antes. El descubrimiento se anunció en una brevísima nota en los Comptes Rendus de la Academia de Ciencias de París, aunque la descripción formal del nuevo género y especie (T. pachecoi) apareció en una publicación del propio Museo de Sabadell ${ }^{18}$. En ambos trabajos, los dos paleontólogos catalanes firmaron junto al especialista francés René Lavocat (1909-2007),

14. Crusafont, Miquel. Copia de carta a George G. Simpson. 23 May 1946. AMC, doc. 045b/CProf/4529

15. Crusafont Pairó, Miguel; Fernández de Villalta, José; Bataller, José Ramón. Los castores fósiles de España. Boletín del Instituto Geológico y Minero de España. 1948; 61: 319-449.

16. Simpson, George Gaylord; Roe, Anne. Quantitative zoology. New York: McGraw-Hill; 1939.

17. Agustí, n. 5, p. 32

18. Fernández de Villalta, José; Crusafont, Miguel; Lavocat, René. Découverte en Europe de ruminants fossiles à corne occipitale. Comptes Rendus Hebdomadaires des Séances de l'Académie des Sciences. 1946a; 222: 406; Fernández de Villalta, José; Crusafont, Miguel; Lavocat, René. Primer hallazgo en Europa de Rumiantes fósiles tricornios. Publicaciones del Museo de Sabadell, Comunicaciones Científicas en Paleontología. 1946b: 1-4. 
con quien Crusafont se había encontrado en París en el curso del viaje que éste realizó por Francia y Suiza en ese mismo año, gracias a la concesión de una beca por parte del Consejo Superior de Investigaciones Científicas ${ }^{19}$.

Triceromeryx no solamente era un ser fascinante por sus características anatómicas - mostraba dos protuberancias óseas frontales, a la manera de las jirafas, más una tercera occipital que, además, estaba bifurcada-, sino que planteaba problemas evolutivos y biogeográficos muy interesantes. Efectivamente, el animal evocaba a las conocidas especies norteamericanas del género Cranioceras, que sin embargo no se daban en Europa. ¿Sería Triceromeryx el resultado de una emigración, o un caso de convergencia? Crusafont y Villalta pidieron a Simpson su opinión al respecto ${ }^{20}$. Este no dudó en considerar el hallazgo del nuevo género como «one of the most remarkable among the many that you have made». Menos seguridad mostraba en dar una respuesta a la cuestión planteada, entre otras cosas, por no ser especialista en tales mamíferos; no obstante, se mostraba proclive a un parentesco estrecho entre ambos géneros antes que a una convergencia ${ }^{21}$. De todos modos, le pasó la información a un experto en estos artiodáctilos, Ruben A. Stirton (1901-1966), profesor en la Universidad de California, cuya respuesta, más prudente todavía, tramitó el propio Simpson a los catalanes $^{22}$. Triceromeryx todavía fue el tema de unas cuantas cartas más, en las que los catalanes se iban decantando por la explicación basada en la convergencia y en una relación con los jiráfidos, en buena medida coincidente con la opinión que Stirton había formulado ${ }^{23}$. Ya en los cincuenta, el asunto seguía abierto y otro especialista, el sueco Birger Bohlin (1898-1990), criticaba la hipótesis de la convergencia y se inclinaba por el parentesco con Cranioceras $^{24}$. Simpson volvió a terciar, adoptando una postura en cierto modo intermedia, pero en todo caso contraria a la pretensión de Crusafont de marcar radicalmente la peculiaridad de Triceromey $x^{25}$.

\footnotetext{
19. Mañosa, n. 1, p. 1455.

20. Crusafont, Miquel; Fernández de Villalta, José. Copia de carta a George G. Simpson. 5 Jul 1946. AMC, doc. 045b/CProf/4546.

21. Simpson, George G. Carta a Miquel Crusafont y José Fernández de Villalta. 9 Oct 1946. AMC, doc. 045b/CProf/4567.

22. Simpson, George G. Carta a Miquel Crusafont y José Fernández de Villalta. 28 Oct 1946. AMC, doc. 045b/CProf/4571.

23. Crusafont, Miquel; Fernández de Villalta, José. Copia de cartas a George G. Simpson. 17 Nov 1946, 20 Feb 1947. AMC, doc. 045b/CProf/4576, 045b/CProf/4610.

24. Crusafont, Miquel. Copia de Carta a George G. Simpson. 5 Feb 1953. AMC, doc. 045c/CProf/4956.

25. Simpson, George G. Carta a Miquel Crusafont. 11 Feb 1953. AMC, doc. 045c/CProf/4963.
} 
El trabajo de Crusafont y Villalta había empezado de este modo a circular a finales de los cuarenta por la comunidad internacional de paleontólogos, gracias tanto a la propia acción difusora emprendida por ellos, como al apoyo que les empezaban a ofrecer personajes como Simpson. Pero no todo era relación estrictamente científica. Para esa época, Crusafont ya se sentía suficientemente confiado como para solicitar de Simpson una intermediación para un asunto personal. Crusafont, aquejado de una úlcera duodenal, era sabedor de que un especialista de origen chino afincado en Nueva York pretendía haber desarrollado un tratamiento eficaz. Recurrió entonces a Simpson por ver si le podía facilitar algún tipo de información o contacto, cosa que, tras varios intentos, consiguió ${ }^{26}$.

No sabemos si el tratamiento del doctor Co Tui alivió los síntomas de Crusafont. Simpson, desde luego, sí probó su buena disposición hacia un colega que, aislado en un país depauperado y en aquel momento poco relevante en el concierto internacional, parecía querer trabajar bien. Se estaba forjando no solamente una colaboración académica, sino también una genuina relación personal.

\section{Orientaciones y divergencias}

No eran fáciles las condiciones de trabajo de Crusafont y sus colaboradores, entre los cuales ya comenzaba a despuntar su joven paisano Jaume Truyols Santonja (n. 1921). En una carta dirigida a Simpson en 1948 - para entonces ya hacía tiempo que le escribía en castellano, pues Simpson le había dicho que sabía leer dicho idioma sin dificultad ${ }^{27}$-, en la que le pedía que avalara su inscripción y la de Truyols a la Society for the Study of Evolution, editora de la revista Evolution, le adelantaba su intención de preparar un trabajo generalista sobre evolución, con la pretensión de «hacer entrar en España ideas nuevas sobre este apasionante problema». Y añadía, tal vez con un punto de victimismo:

26. Crusafont, n. 14. Simpson, George G. Cartas a Miquel Crusafont. 24 Jun 1946, 1 Oct 1947. AMC, doc. 045b/CProf/4539, 006a/CP/150. Crusafont, Miquel. Copia de carta a George G. Simpson. 5 Nov 1947. AMC, doc. 006a/CP/156. Simpson, George G. Cartas a Miquel Crusafont. 18 Nov 1947, 28 Nov 1947. AMC, doc. 065b/Cacad/9108, 006a/CP/159.

27. Simpson, George G. Carta a Miquel Crusafont y José Fernández de Villalta. 13 Mar 1947. AMC, doc. 065b/Cacad/9009. 
«Aquí he tr[abajado] sin ninguna clase de escuela, pues en España puede decirse que nada se ha hecho dentro de este campo. Por ello se explica que uno sienta esta clase de inseguridades. Perdóneme que quizás me exprese con excesiva franqueza pero lo hago confiando en que Vd. se hará cargo de lo que significa tener que trabajar en estas condiciones y además porque le admiro y quisiera poder algún día entrar en esa magnífica y gran comunidad de las personas devotas de la ciencia apasionante de la Paleontología y sus derivados» 28 .

Puede dudarse de que la modestia de Crusafont llegara al punto de no verse todavía miembro de esa «comunidad» paleontológica a la que aludía. En cualquier caso, sí estaba preocupado por no cumplir con lo que sería un estándar profesional en lo que respecta a su formación académica -con las consecuencias de cómo podría ser visto por los colegas-, si atendemos a lo que sostiene Mañosa ${ }^{29}$, quien recuerda que en ese mismo año de 1948 Crusafont se licenció en Ciencias Naturales en Madrid, para doctorarse dos años después con una tesis sobre Los jiráfidos fósiles de España ${ }^{30}$. En buena medida, era la forma de reafirmar su vocación por la paleontología desde la reivindicación de una profesionalidad genuina. Crusafont debió de sentirse muy complacido cuando en 1953, en respuesta a una carta que no ha podido ser localizada, Simpson le decía que «You are certainly more than qualified for membership in the Society of Vertebrate Paleontology», al tiempo que le anunciaba que él mismo lo iba a nominar para su ingreso ${ }^{31}$.

Crusafont, en todo caso, estaba dispuesto a hacerse un nombre no solamente como paleontólogo, sino también como estudioso de la evolución. Agustí ha apuntado que la formación como farmacéutico de Crusafont le hizo ser más sensible al componente biológico de la paleontología de lo que era habitual entre los estudiosos de los fósiles en España, más inclinados a lo propiamente geológico o estratigráfico ${ }^{32}$. Ciertamente, la mayoría de los paleontólogos españoles, hasta esa época, respondían a tal perfil, y alguna excepción notable, como la que representó Eduardo Boscá (1843-1924) con

28. Crusafont, Miquel. Carta a George G. Simpson. 11 May 1948. American Philosophical Society (APS); George Gaylord Simpson Papers (GGSP), serie I, caja 17.

29. Mañosa, n. 1, p. 1455-1456. Por su parte, Truyols 1986, n. 3, p. 34, indica que «alguien» habría insinuado que Crusafont era un simple aficionado.

30. Fue publicada en 1952; Crusafont, Miquel. Los Jiráfidos fósiles de España. Memorias y Comunicaciones del Instituto Geológico de la Diputación Provincial de Barcelona. 1952; 8: 15-239.

31. Simpson, George G. Carta a Miquel Crusafont. 19 Oct 1953. AMC, doc. 045c/CProf/5012.

32. Agustí, n. 5. 
sus estudios paleobiológicos sobre los fósiles argentinos de la colección donada por José Rodrigo Botet a la ciudad de Valencia, obedecía así mismo a una reconversión desde especialidades naturalistas claramente biológicas ${ }^{33}$. Incluso paleontólogos declaradamente evolucionistas, como José Royo Gómez (1895-1961), apenas introducían interpretaciones biológicas en sus estudios, fuera de algunas contribuciones expresamente divulgativas ${ }^{34}$.

Crusafont, desde luego, vivía ya en una época donde ser paleontólogo implicaba necesariamente ser evolucionista. Esto, que no suponía tensión alguna en la mayor parte del mundo, no dejaba de ser problemático, sin embargo, en aquella España de la posguerra civil, con un estado nacionalcatólico que había permitido una revivificación inusitada de posturas antievolucionistas $^{35}$. Ya hemos comentado que el propio Crusafont era un católico fervoroso, aunque estaba lejos de comulgar con otras máximas ideológicas del régimen franquista. Su actitud, por tanto, partía, como la de tantos otros, de conciliar su fe con la asunción de la evolución de la vida ${ }^{36}$.

Las propuestas de diferentes autores, sobre todo la del italiano Alberto Carlo Blanc (1906-1960), con su teoría de la cosmolisis, según la cual existía una tendencia evolutiva hacia la segregación de un tipo ancestral sintético en tipos particulares, y, desde luego, la del jesuita francés Pierre Teilhard de Chardin (1881-1955), con su asunción de una ortogénesis «de fondo»-que marcaría la tendencia de los seres vivos a aumentar su nivel de complejidad y de conciencia-, tenían por aquellas décadas centrales del siglo XX suficiente predicamento como para circular ampliamente, a pesar de su heterodoxia respecto a la cada vez más asentada nueva síntesis. Crusafont las asumió en buena medida, en esa línea de lo que Agustí, tomándolo del propio Crusafont, ha denominado «evolucionismo meridional», caracterizado por un eclecticismo frente a la oposición tradicional darwinismo-lamarckismo, y un declarado carácter finalista coherente con una asunción teísta ${ }^{37}$. La

33. Catalá, Jesús I. El desarrollo de una carrera científica en un contexto institucional precario: el caso del naturalista Eduardo Boscá Casanoves (1843-1924). Cronos. 2004; 7: 3-60.

34. Royo Gómez, José. La Paleontología y la evolución de las especies. Conferencias y Reseñas Científicas de la Real Sociedad Española de Historia Natural. 1927; 2: 189-205.

35. Blázquez, 2009, 2011, n. 9. Catalá, Jesús I. Spain and Portugal. In: Blancke, Stefaan; Hjermitslev, Hans Henrik; Kjærgaard, Peter C., eds. The history of creationism in Europe. Baltimore: Johns Hopkins University Press; 2013, en prensa.

36. Intención, en todo caso, que no siempre le fue fácil llevar adelante; véase al respecto el artículo de Florensa en este mismo monográfico de Dynamis.

37. Agustí, n. 5, p. 29; Pelayo, n. 8, p. 124. 
direccionalidad de que hacía gala la propuesta teilhardiana, y la que se le imputó - pese a que Blanc nunca se manifestó favorable- a la cosmolisis, se avenían bien con los fundamentos doctrinales de la fe católica.

Crusafont supo manejarse en ese terreno, y fue capaz de mantener con Simpson una relación científica más que provechosa sin llegar nunca a claudicar ante el manifiesto materialismo neodarwinista de éste, ni a dejarse «contagiar» por su rechazo a todo tipo de finalismo ${ }^{38}$. En buena medida, esto fue posible porque Crusafont, con el auxilio de Truyols, se mostró como un especialista avezado en esa paleontología de base cuantitativa que autores como el finés Björn Kurtén (1924-1988) o el propio Simpson estaban promoviendo. A comienzos de los años cuarenta, en plena génesis de la síntesis evolucionista, Simpson no dudó en apoyar desde su enfoque paleontológico la proclama de mensurabilidad de la selección natural que, desde la genética, había adelantado Theodosius Dobzhansky. El enfoque cuantitativo, pensaba él, permitiría a la paleontología librarse de la carga nada halagüeña y preñada de metafísica de la evolución dirigida ${ }^{39}$, por entonces consustancial a cualquier aproximación al hecho evolutivo que se hiciera a partir de fósiles. Este prurito también tendría un estímulo, como señalan otros autores, basado en emular los enfoques avanzados, de base matemática, de la propia genética, asumiendo que la paleontología, en todo caso, nunca podría exhibir un enfoque experimental ${ }^{40}$. En todo caso, Simpson se esforzó por hacer de la paleontología una ciencia biológica más; ya en el título de Quantitative zoology - pero también en muchos pasajesprocura desdibujar los límites entre ciencia de los fósiles y ciencia de los vivientes, de modo que las interpretaciones teóricas basadas en los fósiles han de aspirar a ser equivalentes a aquellas derivadas del estudio de seres vivos actuales ${ }^{41}$. Seguramente, tanto las prevenciones ideológicas como la congruencia con otras tradiciones aplicadas al estudio de la evolución pesaron en el ánimo de Simpson y de muchos otros paleontólogos «cuantitativos»; y también pudo hacer algo al respecto cierto anhelo de respetabilidad, que un indumento matemático podía saciar. Crusafont, de hecho, buscó más la

38. Véase para más detalle Glick, Thomas F. Miquel Crusafont, Teilhard de Chardin and the reception of the Synthetic theory in Spain. In: Engels, Eve-Marie; Glick, Thomas F., eds., The reception of Charles Darwin in Europe. London/New York: Continuum; 2008, vol. 2, p. 553-568.

39. Smocovitis, Vassiliki Betty. Unifying biology: The evolutionary synthesis and evolutionary biology. Princeton: Princeton University Press; 1996, p. 135.

40. Agustí, n. 5, p. 32.

41. Laporte, n. 7, p. 27-28. 
respetabilidad que no la liberación metafísica para la paleontología evolutiva; pretendió encontrar en sus estudios «masterométricos» una confirmación a sus ideas de evolución finalista. Las discusiones con Simpson, en este terreno, fueron inevitables y adquirieron tonos realmente apasionados en algunos momentos.

La primera declaración de principios evolucionistas de Crusafont vino con el envío a Simpson de ese trabajo al que aludíamos al comienzo de este apartado, y que llevaba un título tan significativo como Concepciones cosmovitalistas del evolucionismo ${ }^{42}$, en el que ensayaba una propuesta ecléctica entre lamarckismo, mutacionismo y darwinismo, aunque con preeminencia del primero ${ }^{43}$. En la correspondencia conservada no hay referencias a la impresión que causó en Simpson, aunque Crusafont parece que pretendía condicionar la posible respuesta adversa, al presentárselo como un producto que no tenía «nada de particular» y que, a la postre, no era sino un intento divulgativo dirigido a un país que llevaba «medio siglo» de retraso en el tema ${ }^{44}$. Simpson sí se pronunció rotunda y sinceramente años después, en 1955, respecto a la asunción por parte de Crusafont de las ideas de Teilhard y al modo en que interpretaba los enfoques de él mismo:

«Of course as you know I disagree rather radically with your views on evolution, and I find that your brief reference to my views is not entirely in accordance with what I really think about the matter of macroevolution, but I certainly respect your opinions highly and find them of real interest» ${ }^{45}$.

Años después, Crusafont se pronunciaría con no menor sinceridad sobre las críticas de Simpson a una de las obras capitales de Teilhard, Le phénomène humain:

«Realmente me doy cuenta, al leer su crítica, de cuán separados estamos en nuestro enfoque de la cuestión cuando tratamos de llegar a una proyección de nuestros conocimientos hacia la esfera de la interpretación. Dice Vd. que aunque la evolución sea «direccional», como puede ser todo proceso histórico, es en realidad multidireccional y que la línea humana es

42. Crusafont, Miquel. Concepciones cosmovitalistas del evolucionismo. Sabadell: Museo de Sabadell; 1948 (Comunicaciones Científicas en Paleontología; 4).

43. Agustí, n. 5, p. 31

44. Crusafont, Miquel. Copia de cartas a George G. Simpson. 6 Ago 1948, 18 May 1949. AMC, doc. 065c/Cacad/9205, 045c/CProf/4780.

45. Simpson, George G. Carta a Miquel Crusafont. 15 Nov 1955. AMC, doc. 066b/Cacad/9869. 
una de tantas direcciones. Para el P. Teilhard, y así lo veo yo, es cierto que existen multitud de direcciones, pero una sola es totalmente (totalmente en el sentido relativo de la superioridad del hombre) prosperante en cuanto a mayor complejidad-consciencia» ${ }^{46}$.

Lo que fascinaba a Crusafont era pensar, de acuerdo con Teilhard, que hasta las fronteras de lo humano la evolución actuaba de forma divergente, mientras que una vez superada la mera animalidad, la evolución convergía hacia el hombre. Y, de alguna manera, le dolía que su amigo Simpson - para entonces ya se habían conocido personalmente-, al rehuir dar un paso metafísico en sus indagaciones, cayera a su juicio en otra metafísica, materialista eso sí, que postulaba una materia autosuficiente. En todo esto no había, en realidad, nada que no se hubiera planteado ya en los viejos debates de los albores del darwinismo, y aun antes. Pero Teilhard le daba a Crusafont la coartada de su condición de paleontólogo. No era un mero teólogo, ni siquiera un filósofo, sino un hombre de ciencia, de ciencia natural, en el sentido ya entonces habitual del término. Y era discutido por los hombres de ciencia en sus foros, como demostraba la propia crítica de Simpson. La actitud de éste, sin embargo, era diferente, y no solo por no asumir las tesis del jesuita francés, sino por ser tajante en la separación entre sus obras paleontológicas y biológicas y sus escritos «metafísicos». De hecho, su dimisión del comité para la publicación de las obras de Teilhard, fue el resultado de que «even now after so many years, not a single one of Father Teilhard's scientific works has been included in the collection» ${ }^{47}$.

Crusafont, miembro de ese mismo comité, lamentó desde luego la dimisión de Simpson, aunque reconocía que los argumentos dados eran lógicos. En la misma carta donde le expresaba esto, añadía:

«Vd. ya sabe que en el fondo de nuestros conceptos puramente racionales, estamos de acuerdo. Lo único que nos separan son nuestras extrapolaciones hacia la metafísica. De todos modos parece ser que la Mecánica estadística hoy señala el carácter improbabilístico y con tendencia convergente y por ello indicando un télos en el bloque espacio-tiempo, de los sistemas vitales, en contraposición a la divergencia de los sistemas inorgánicos. Esto parece reforzar la posición finalística. Es difícil para mí concebir todo lo que existe

\footnotetext{
46. Crusafont, Miquel. Carta a George G. Simpson. 11 Ago 1961. APS; GGSP, serie I, caja 17. Énfasis en el original.

47. Simpson, George G. Copia de Carta a Miquel Crusafont. 18 Mar 1964. APS; GGSP, serie I, caja 17.
} 
sin un alfa y un omega: no me parece lógico que exista algo por sí mismo y que no se halle un principio y un fin para todas las cosas existentes. Perdóneme mi insistencia» ${ }^{48}$.

Casi hay angustia en tan premiosas frases. Una expresión de acuerdo, difícilmente sostenible, deja paso a un intento de hallar una justificación en la ciencia natural, para rendirse inmediatamente al reconocimiento de que hay algo más en la postura existencial asumida. Y al final, un ruego casi vergonzante de disculpa. No era la primera vez, en todo caso, que cierto sentimiento de inferioridad afloraba en las cartas de Crusafont a Simpson. En la ya citada en que contestaba a la crítica de Simpson a Le phénomène humain, se despedía del siguiente modo:

«Vd. me perdonará mi atrevimiento en hacerle perder tanto tiempo. A mí todo esto me preocupa y me interesa. Quizás me encuentre Vd. "naif" pero yo le hablo con toda sinceridad y afecto. Incluso quizás me considere Vd. escasamente inteligente. Pero le aseguro que procuro buscar respuestas» ${ }^{49}$.

\section{La proyección internacional}

No cabe duda de que Crusafont apreciaba a Simpson no solamente como científico, sino también como persona. Pero hubo también, desde luego, un interés en el cultivo de la relación por parte del catalán. Simpson, en cierto modo, acabó por erigirse en un verdadero agente para la promoción internacional de la obra de Crusafont y su núcleo de colaboradores.

Las primeras publicaciones fuera de España que rindió Crusafont, siempre compartidas con Fernández de Villalta, aparecieron en Francia entre 1946 y 1948. Además de la ya citada sobre el descubrimiento de Triceromeryx en los Comptes Rendus de la Academia de Ciencias, destaca la serie de ocho notas breves publicadas en los Comptes rendus sommaires de la Société Géologique de France, en las que se iba dando noticia de diferentes yacimientos de mamíferos del Neógeno de varias cuencas sedimentarias de

48. Crusafont, Miquel. Carta a George G. Simpson. 31 Mar 1964. APS; GGSP, serie I, caja 17. Énfasis en el original.

49. Crusafont, Miquel. Carta a George G. Simpson. 11 Ago 1961. APS; GGSP, serie I, caja 17. Énfasis en el original. 
España $^{50}$. A todo ello habría que añadir un artículo en el que se describía una nueva especie de erizo fósil, Palerinaceus vireti, que apareció también en francés pero en una revista suiza ${ }^{51}$. El contacto con colegas franceses y suizos, establecido por Crusafont en el ya aludido viaje que llevó a cabo en 1946, más el buen conocimiento que tenía de la lengua francesa, hacían de estas revistas una opción muy razonable para buscar una mayor difusión a sus trabajos, hasta entonces constreñidos a medios españoles. En conjunto, se trataba de aportaciones todavía fuertemente ligadas a consideraciones típicamente estratigráficas y de descripción sistemática, aunque, como bien argumenta Truyols ${ }^{52}$, con modos de trabajo diferentes a los que habían venido desarrollándose en los años anteriores a la Guerra Civil por parte de los especialistas autóctonos en vertebrados fósiles.

Crusafont, en todo caso, pensó que sería conveniente publicar en inglés y en los Estados Unidos. Además, había descrito un nuevo género, Triceromeryx, que por los problemas de filiación que planteaba resultaría a priori de gran interés para los paleontólogos norteamericanos; no en balde, lo que se tenía que resolver era su relación con un grupo genuino del Nuevo Mundo, el que representaba Cranioceras y sus formas afines. La confianza creciente que iba ganando en su relación con Simpson le llevó a plantearle en marzo de 1948 la posibilidad de escribir una nota sobre su hallazgo en American Museum Novitates, la revista de la institución donde éste trabajaba ${ }^{53}$. Simpson arguyó que la revista solamente admitía trabajos basados en estudios llevados a cabo en el propio Museo; como alternativa, le señaló American Journal of Science, revista de la que era associate editor. Incluso, se ofreció a traducirle el manuscrito al inglés ${ }^{54}$. Ante tal propuesta, no es extraño que Crusafont aceptara, de modo que se comprometió a redactar un trabajo en el que pretendía ofrecer una descripción detallada de la osteología de Triceromeryx, más algunas inferencias sobre taxonomía

50. Fernández de Villalta, José; Crusafont, Miquel. 1946-48. Les gisements de Mammifères du Néogène espagnol. Comptes Rendus Sommaires de la Société Géologique de France. 1946; 49-51,133-134, 224-227. 1947; 28-30, 256-268, 278-280. 1948; 167-170, 186-188.

51. Crusafont, Miquel; Fernández de Villalta, José. Sur un nouveau Palerinaceus du Pontien d'Espagne. Eclogae Geologicae Helvetiae. 1947; 40: 320-333.

52. Truyols, n. 3, p. 22.

53. Crusafont, Miquel. Carta a George G. Simpson. 5 Mar 1948. APS; GGSP, serie I, caja 17.

54. Simpson, George G. Carta a Miquel Crusafont. 16 Mar 1948. AMC, doc. 065c/Cacad/9205. 
y filogenia ${ }^{55}$. Quería mostrarse al mismo tiempo como un paleontólogo riguroso en su trabajo descriptivo e interesado por las cuestiones teóricas.

Crusafont, sin embargo, no publicó ningún artículo en American Journal of Science. En la correspondencia aparecen disculpas y anuncios de un envío próximo ${ }^{56}$, pero lo cierto es que el trabajo nunca apareció. Por aquellos años de finales de la década de los cuarenta, Crusafont estuvo preparando una comunicación sobre zoogeografía de los mamíferos del Neógeno de España para el XIII Congreso Internacional de Zoología de 1949, aunque cuestiones financieras le impidieron desplazarse a París, la ciudad que acogía la reunión ${ }^{57}$. Se encontraba también ultimando su tesis doctoral, en la cual Triceromeryx era asunto fundamental. Y, respecto a éste, las dudas propias y ajenas que ya hemos comentado le estarían probablemente haciendo ver que había que ser cauto con ciertas opiniones. Tal vez esto explique la razón última de que no redactara el trabajo para la revista estadounidense, pese a las facilidades que se le estaban dando. La correspondencia, algo fragmentaria en lo que nos ha llegado para esa época, no permite ofrecer una interpretación más segura.

Triceromeryx, por otro lado, era un tema compartido con Fernández de Villalta. Aunque al binomio aún le quedaba un buen puñado de colaboraciones por firmar en los años siguientes y hasta mitad de la década de los cincuenta, Crusafont estaba en cualquier caso incorporando a su quehacer científico a nuevos colaboradores, entre ellos, desde 1947, al ya mencionado Truyols $^{58}$. Sería con éste con quien, a la postre, firmaría la mayoría de sus trabajos en inglés y algunos de los que, desde el punto de vista teórico, mostraron mayor enjundia. Ya en 1953 publicaron en España un artículo, «Un ensayo goniométrico sobre la carnicera inferior de los Fisípedos» ${ }^{59}$, en el que iniciaban lo que sería una línea muy fecunda de estudio de la evolución de los carnívoros que se prolongaría durante más de un decenio.

\footnotetext{
55. Crusafont, Miquel. Carta a George G. Simpson. 17 Abr 1948. APS; GGSP, serie I, caja 17.

56. Crusafont, Miquel. Carta a George G. Simpson. 11 May 1948. APS; GGSP, serie I, caja 17. Crusafont, Miquel. Copia de cartas a: George G. Simpson. 6 Ago 1948, 18 May 1949. AMC, doc. 065c/ Cacad/9205, 045c/CProf/4780.

57. Crusafont, Miquel. Carta a George G. Simpson. 6 Ago 1948. AMC, doc. 065c/Cacad/9205.

58. Agustí, Jordi. L'obra paleontològica de Miquel Crusafont. Ressenya bibliográfica i trets fonamentals. Arraona. 1994; 14: 105-108 (106).

59. Crusafont, Miquel; Truyols, Jaume. Un ensayo goniométrico sobre la carnicera inferior de los fisípedos. Estudios Geológicos. 1953; 18: 225-254.
} 
Aunque no firmó el trabajo de 1948 sobre los castores, Truyols ya era mencionado en los agradecimientos de la parte de biometría por Crusafont, por haber «facilitado la confección de estadísticas y gráficos» ${ }^{60}$. De hecho, Truyols fue durante este período el colaborador específico en los estudios biométricos (también en los biogeográficos) de Crusafont, mientras que Villalta era el de las contribuciones descriptivas faunísticas ${ }^{61}$. Ya hemos visto, por otro lado, cómo Crusafont pidió a Simpson que avalara su ingreso y el de Truyols en la Society for the Study of Evolution. En el mismo año de 1948, Crusafont procuró dar una difusión añadida a los trabajos biométricos con una reseña en el Boletín de la Real Sociedad Española de Historia Natural ${ }^{62}$, en el mismo volumen, por cierto, en que otro destacado paleontólogo español, Bermudo Meléndez (1912-1999), se ocupaba, en sendas notas, de la paleontología en relación con la nueva síntesis y del evolucionismo $«$ regresionista ${ }^{6}$.

Crusafont ya había barruntado publicar algo en Evolution allá por 1950. El trabajo iba a tratar sobre la filogenia de los jiráfidos, su grupo predilecto por entonces. Creía, no obstante, que la revista admitía trabajos en español ${ }^{64}$, hasta que Simpson le aclaró que solamente el inglés estaba contemplado ${ }^{65}$. Crusafont tampoco fue esta ocasión más allá de la declaración de intenciones. Aquellos primeros años de la década de los cincuenta, por otro lado, fueron de cierto enfriamiento de la relación con Simpson; lagunas de conservación aparte, el intercambio epistolar registra una clara disminución entre 1950 y 1955. En el ínterin fue cuando Crusafont

60. Fernández de Villalta; Crusafont; Lavocat, 1946a, n. 18, p. 110.

61. Agustí, n. 58, p. 107.

62. Crusafont, Miquel. Reseñas Científicas. Primeras aplicaciones de la biometría paleontológica en España. Boletín de la Real Sociedad Española de Historia Natural (Sección Geológica). 1948; 46: 285-288.

63. Meléndez, Bermudo. Reseñas Científicas. La Paleontología ante las nuevas tendencias de «síntesis» neo-darwinistas. Boletín de la Real Sociedad Española de Historia Natural (Sección Geológica). 1948; 46: 143-151. Meléndez, Bermudo. Reseñas Científicas. Sobre el evolucionismo regresionista. Boletín de la Real Sociedad Española de Historia Natural (Sección Geológica). 1948; 46: 279-284. Sobre la oposición de Meléndez a la nueva síntesis, véase Blázquez, Francisco. La teoría sintética de la evolución en España. Primeros encuentros y desencuentros. Llull. 2001; 24: 289-313.

64. Crusafont, Miquel. Copia de carta a George G. Simpson. 30 Abr 1950. AMC, doc. 065d/Cacad/9374.

65. Simpson, George G. Carta a Miquel Crusafont. 21 Ago 1951. AMC, doc. 065d/Cacad/9493. Por cierto, que la justificación que ofrecía Simpson nada tenía que ver con criterios de difusión: «The journal has not been publishing articles in languages other than English because of the very great expense of having other languages type-set in this country». 
y Truyols publicaron su «Ensayo goniométrico», un trabajo riguroso pero en todo caso concebido más desde el valor descriptivo de la biometría que no desde el punto de vista de la interpretación evolutiva ${ }^{66}$.

Fue en noviembre de 1955 cuando Crusafont anunció a Simpson que Truyols y él tenían previsto enviar en un plazo de dos semanas un artículo a Evolution. Decía que lo enviarían ellos mismos en inglés, pero le pedía que fuera él quien lo presentara tras haberlo revisado ${ }^{67}$. Simpson respondió que podían enviarlo directamente al editor, a la sazón Everett C. Olson (1910-1993) ${ }^{68}$, por entonces en la Universidad de Chicago; además, volvió a ofrecerse a traducir el trabajo del castellano al inglés ${ }^{69}$. El 29 de diciembre, Crusafont envió a Simpson el manuscrito ${ }^{70}$; éste, que acababa de ser intervenido quirúrgicamente, tenía lista la traducción a finales del mes de enero de 1956. Simpson juzgó que el artículo «is certainly a valuable one», si bien no negaba que «I do disagree with some points of your interpretation». En todo caso, no entró en la cuestión. Lo que sí requirió de los catalanes fue la redacción del preceptivo resumen y la inclusión de las citas bibliográficas de varios autores que eran simplemente mencionados en el texto ${ }^{71}$. Por una carta de Simpson (las de Crusafont de 1956, desgraciadamente, no se han conservado), sabemos que el 7 de febrero ya habían sido mandadas las adiciones y correcciones solicitadas. Tanto celo se tomó Simpson, que incluso mandó fotografiar las ilustraciones que acompañaban al manuscrito como medida de precaución, aunque también, según reconocía, porque había pensado usarlas para sus clases de evolución de los carnívoros en Columbia $^{72}$. El 27 de febrero, Simpson volvió a escribir a Crusafont para comunicarle que Olson había encontrado el artículo «extremely interesting» y que, efectivamente, había sido aceptado para su publicación ${ }^{73}$. De este

\footnotetext{
66. Agustí, n. 5, p. 32-34.

67. Crusafont, Miquel. Carta a George G. Simpson. 11 Nov 1955. APS; GGSP, serie I, caja 17.

68. Olson era un paleontólogo de la onda de Simpson que al frente de la revista estaba realizando una importante labor de cara a la incorporación de la comunidad paleontológica a la nueva síntesis; véase Laporte, n. 7, p. 34.

69. Simpson, nota 45.

70. Simpson, George G. Copia de carta a Miquel Crusafont. 11 Ene 1956. APS; GGSP, serie I, caja 17.

71. Simpson, George G. Copia de carta a Miquel Crusafont. 26 Ene 1956. APS; GGSP, serie I, caja 17.

72. Simpson, George G. Copia de carta a Miquel Crusafont. 17 Feb 1956. APS; GGSP, serie I, caja 17.

73. Simpson, George G. Copia de carta a Miquel Crusafont. 27 Feb 1956. APS; GGSP, serie I, caja 17.
} 
modo, «A biometric study of the evolution of Fissiped Carnivores» apareció en el fascículo 3 del volumen de aquel año ${ }^{74}$.

En el artículo, Crusafont y Truyols desarrollaron el método denominado «masterometría», que permitía medir los dientes de los carnívoros de un modo que facilitaba la comparación y la estimación de los cambios en una línea evolutiva. A partir de su aplicación, derivaban unas conclusiones que favorecían una visión ortogenética ${ }^{75}$. No entraremos en el contenido técnico, toda vez que Agustí ya lo ha estudiado muy bien en una de sus contribuciones. Este mismo autor, en todo caso, plantea en el mismo trabajo una pregunta fundamental: ¿cómo fue posible que un artículo contaminado de ortogénesis y finalismo fuera publicado en Evolution, órgano consagrado de los partidarios de la síntesis neodarwinista? Él mismo aventura que el enfoque cuantitativo aportado, que, lejos de reproducir las propuestas de Simpson u otros autores, era ciertamente original, impresionaría favorablemente al consejo editor ${ }^{76}$. Aquí, sin rechazar esa explicación, nos permitimos conjeturar que la intervención de Simpson pudo ser determinante. En una de las cartas, Simpson le decía a Crusafont:

«I have of course personally recommended its publication but the decision will rest with the editorial board. No doubt you will hear direct from Dr. Olson as to that» ${ }^{77}$.

A pesar de la proclama enfática sobre la independencia del comité editorial, ya hemos visto quién adelantó a Crusafont la noticia de la aceptación. Simpson, no hay que olvidarlo, había jugado un papel decisivo en el arranque de Evolution y en la fundación de la Society for the Study of Evolution, de la que fue primer presidente, cargo desde el cual procuró dotar de financiación a la revista en sus inicios ${ }^{78}$. En ese sentido, no debería extrañar que, al margen de formalidades, su opinión fuera tenida en consideración.

Simpson, de todos modos, no quería dar pie a que alguien pensara que había tenido algo que ver con el artículo de Crusafont y Truyols. Olson propuso añadir una nota sobre la traducción, para decir que había sido

74. Crusafont, Miquel; Truyols, Jaume. A biometric study of the evolution of Fissiped Carnivores. Evolution. 1956; 10: 314-332.

75. Glick, 1994, n. 6, p. 50.

76. Agustí, n. 5, p. 34.

77. Simpson, n. 73.

78. Laporte, n. 7, p. 34. 
revisada y aceptada por los autores. Pero Simpson le rogó que su nombre no apareciera:

«I have requested that my name should not appear as translator of the paper, because that is a pleasant task for which I neither deserve nor desire credit and because I am afraid that if I were named as translator some of the readers of EVOLUTION might think that I had something to do with the writing of the paper or with the ideas expressed therein» ${ }^{79}$.

Una cosa era dar apoyo, y otra bien distinta dar que pensar acerca de que uno de los principales proponentes de la nueva síntesis coqueteara con teorías finalistas. De todos modos, el artículo no solamente recibió los parabienes de autores a priori más próximos, como fue el caso de Blanc, sino también de verdaderos puntales de la nueva síntesis. Concretamente, Julian Huxley (1887-1975) halló en la masterometría un apoyo a sus ideas sobre la estasigénesis o «evolución horizontal». Crusafont le hizo llegar, incluso, datos inéditos añadidos. Se conocieron personalmente poco después, en mayo de 1957, con ocasión de una reunión en homenaje a Linneo que tuvo lugar en Uppsala, y allí discutieron sobre el asunto ${ }^{80}$.

Esta nueva conexión también fue explotada provechosamente por Crusafont en el proceso de internacionalización de sus aportaciones. En 1958, éste y Truyols firmaron una nueva nota, «A quantitative study of stasigenesis in Fissiped Carnivores», publicada nada menos que en Natu$r e^{81}$. La traducción corrió a cargo de Huxley, según le contó a Simpson, al tiempo que le decía estar preparando un nuevo artículo para Evolution, cuya traducción pensaba encomendar ahora a Kurtén «con el objeto de no molestarle a Vd. en las actuales circunstancias» ${ }^{82}$. Simpson estaba por entonces - finales de 1957- recuperándose del gravísimo accidente que había sufrido en el verano de 1956, cuando le cayó un árbol en el transcurso de una expedición en la Amazonia brasileña. Además de las consecuencias físicas, el accidente le dejó secuelas psíquicas considerables ${ }^{83}$.

\footnotetext{
79. Simpson, n. 73. Mayúsculas en el original.

80. Crusafont, Miquel. Carta a George G. Simpson. 18 Mar 1957. APS; GGSP, serie I, caja 17. Crusafont, Miquel. Copia de carta a George G. Simpson. 6 Jun 1975. AMC, doc. 051a/Cprof/8176. Véase también Glick, 1994, n. 6, p. 50.

81. Crusafont, Miquel; Truyols, Jaume. A quantitative study of stasigenesis in Fissiped Carnivores. Nature. 1958; 181: 289-290.

82. Crusafont, Miquel. Carta a George G. Simpson. 30 Dic 1957. APS; GGSP, serie I, caja 17.

83. Laporte, n. 7, p. 249-250.
} 
Lo cierto es que ese nuevo artículo, a la postre, no apareció. Mientras tanto, Simpson, que ya había vuelto a la actividad, le envió a Crusafont una serie de consideraciones críticas sobre su propuesta masterométrica ${ }^{84}$. Éstas fueron muy bien recibidas por el catalán ${ }^{85}$. Simpson, poco después, le envió un trabajo suyo, todavía inédito, sobre la estimación de las semejanzas faunísticas, con la encomienda de que juzgara si era «useful and sound» y le dijera si encontraba «anything objectionable in the references to Crusafont and Truyols» ${ }^{86}$. En cierto modo, Simpson certificaba así una confianza en Crusafont que solamente puede explicarse desde una estimación elevada del quehacer científico de éste.

No tardó en contestar Crusafont, expresándose con notable sinceridad y pidiéndole, ni más ni menos, que un reconocimiento explícito de prioridad:

«Desde luego debe Vd. considerarlo puramente como un ruego y no una obligación, pero toda vez que Vd. ha sido tan bueno que nos ha sometido su trabajo, nosotros le agradeceríamos de corazón tuviera la bondad de indicar que la idea de realizar una comparación a la vez sistemática y ecológica o aun sólo ecológica, y por lo tanto la de hacer entrar el número de individuos, fuimos nosotros los primeros en establecerla y que podía ser interesante» ${ }^{87}$.

Simpson acogió gustosamente todo lo que Crusafont sugería ${ }^{88}$. Su relación científica estaba definitivamente asentada, y la empatía mutua que se profesaban solo requería del encuentro personal para asentarse en una amistad verdadera. Así sucedió con ocasión del viaje a España que Simpson y su esposa hicieron en el verano del año siguiente, es decir, en 1960.

La relación continuó durante dos décadas más, hasta la muerte de Crusafont. La menor intensidad del trabajo científico de este durante los años setenta ${ }^{89}$ condicionó una asiduidad menor en el intercambio epistolar, al tiempo que aumentaba el contenido estrictamente personal de las cartas. Para lo bueno y para lo malo, al final aquellas cartas representaron, sobre todo, la celebración nostálgica de la amistad entre dos ancianos.

84. Simpson, George G. Cartas a Miquel Crusafont. 11 Feb 1959, 19 Mar 1959. AMC, doc. 046b/ Cprof/5549, 046b/Cprof/5571.

85. Crusafont, Miquel. Copia de carta a George G. Simpson. 12 Mar 1959. AMC, doc. 046b/Cprof/5567. Crusafont, Miquel. Carta a George G. Simpson. 25 Mar 1959. APS; GGSP, serie I, caja 17.

86. Simpson, George G. Copia de carta a Miquel Crusafont. 27 Abr 1959. APS; GGSP, serie I, caja 17.

87. Crusafont, Miquel. Carta a George G. Simpson. 2 May 1959. APS; GGSP, serie I, caja 17.

88. Simpson, George G. Copia de carta a Miquel Crusafont. 11 May 1959. APS; GGSP, serie I, caja 17.

89. Acosta, n. 2. 


\section{Conclusión}

En su autobiografía, Simpson definía a Crusafont como «an interesting and admirable man ${ }^{90}$. Admiraba sinceramente su vocación y su empeño para conseguir, en circunstancias tan adversas como las de aquella España, un espacio social digno para la paleontología. Y, desde luego, dio pruebas de admirarlo también como científico.

En los años sesenta, Crusafont y sus colaboradores añadieron muchos más trabajos en el extranjero, preferentemente escritos en francés, a su producción. La proyección internacional de lo que ha venido en llamarse la «escuela paleontológica de Sabadell» ${ }^{91}$ quedaba ya firmemente asentada.

La masterometría dio pie a un nuevo artículo de Crusafont y Truyols en Evolution, ya en el año 1966, en lo que era una respuesta a un artículo del año anterior del propio Simpson, significativamente titulado «A review of masterometry», en el que elaboraba una crítica a la propuesta de aqué$1 \operatorname{los}^{92}$. Las críticas, hasta entonces resueltas básicamente en la privacidad de la correspondencia, tomaban ahora luz pública, sobre todo porque había autores que interpretaban que la masterometría aportaba evidencias contrarias a la teoría sintética, lo cual no podía quedar sin respuesta por parte de Simpson ${ }^{93}$. Mas la amistad entre éste y Crusafont, forjada a lo largo de más de veinte años de relación epistolar y de aquel encuentro episódico de 1960, no se resintió en absoluto.

En aquella relación, ciertamente, el rédito mayor lo obtuvo Crusafont y, con él, la paleontología evolutiva española. Simpson no fue, ni mucho menos, el único apoyo foráneo con que contó Crusafont. Tampoco se puede decir que fuera el más importante, ya que la historia de las otras relaciones científicas mantenidas por Crusafont con otros colegas extranjeros todavía está por escribir. En cualquier caso, sí parece evidente que Simpson fue clave en el modo en que Crusafont se incorporó a la comunidad internacional de paleontólogos. Pasivamente, fue el inspirador de la línea que mayor proyección otorgó a Crusafont. Más activamente, su intervención

90. Simpson, George Gaylord. Concession to the improbable: An unconventional autobiography. New Haven/London: Yale University Press; 1978, p. 189.

91. Mañosa, n. 1.

92. Simpson, George G. A review of masterometry. Evolution. 1965; 19: 249-255. Crusafont, Miquel; Truyols, Jaume. Masterometry and evolution, again. Evolution. 1966; 20: 204-210.

93. Glick, 1994, n. 6, p. 50-51. 
fue crucial a la hora de que el catalán publicara en Evolution, la revista que entonces representaba la punta de lanza de los estudios evolucionistas. Y simbólicamente, el uso efectivo que Simpson hizo de la masterometría, a pesar de las críticas que a la vez lanzó, reforzó extraordinariamente la trayectoria de aquel farmacéutico cuyo trabajo se venía desarrollando en el no demasiado estimulante ambiente que le tocó vivir.

En todo ello, fue importante que Simpson encontrara en Crusafont a un colega genuino, pese a las grandes diferencias que mantenían en cuanto a su idea de la evolución y, en general, a su visión del mundo. Pero también fue decisiva una percepción, más sensible que racional, que Simpson expresó así:

«I can truly say that I have very rarely met anyone who seemed to me a true and valuable friend at first sight as you did and who has continued to mean a great deal to me. There surely is [a] reason why the Spanish word «simpático» is Spanish and does not have an exact equivalent in other languages» ${ }^{94}$.

\section{Agradecimientos}

El autor desea agradecer a Thomas F. Glick, de Boston University, el acceso a la correspondencia de Simpson y Crusafont recogida en este artículo. La edición crítica de estas cartas está actualmente en proceso de preparación por parte del profesor Glick y del autor. 
Miquel Crusafont, George Simpson and the internationalization of evolutionary Palaeontology studies in Spain Jesús I. Catalá Gorgues.......................................

1.- Introduction. 2.-Starting a relationship. 3.-Orientations and differences.

4.--International impact. 5.-Conclusion. 
ABSTRACT: Catalan palaeontologist Miquel Crusafont Pairó (1910-1983) was a leading specialist on fossil mammals of the Iberian Tertiary. He also introduced new quantitative approaches to biological evolution into Spain. Despite many difficulties in carrying out research on evolution in Franco's Spain, Crusafont achieved an outstanding position in the international community of palaeontologists. Besides being a competent scientist, he benefitted from the friendship of American palaeontologist George Gaylord Simpson (19021984), a major proponent of the evolutionary synthesis. This paper explores the relationship between both scientists and stresses the crucial role that Simpson played in the internationalization of Crusafont's work. 